\title{
Recurrent Retinal Detachment After Pars Plana Vitrectomy For Rhegmatogenous Retinal Detachment
}

Yan Fu

Baoding No 1 Central Hospital https://orcid.org/0000-0001-8876-992X

Tian-hao Xie

affiliated hospital of Hebei university

Zhao-Hui Gu (D shzguzhaohui@126.com )

Baoding No 1 Central Hospital

Na Yang

Baoding No 1 Central Hospital

Ren-Fei Geng

Baoding No 1 Central Hospital

Yue-ling Zhang

Baoding No 1 Central Hospital

\section{Research Article}

Keywords: retinal detachment, proliferative vitreoretinopathy, visual outcome, vitrectomy

Posted Date: October 28th, 2021

DOI: https://doi.org/10.21203/rs.2.21163/v2

License: (c) (i) This work is licensed under a Creative Commons Attribution 4.0 International License.

Read Full License 


\section{Abstract \\ Background}

The recurrence of retinal detachment (RD) following rhegmatogenous retinal detachment (RRD) is a relatively common complication that threatens vision and requires further surgery. The purpose of this study was to investigate the risk factors and visual outcomes of recurrent RD following pars plana vitrectomy (PPV) for primary RRD.

\section{Methods}

This was a retrospective follow-up of 343 eyes that underwent initial PPV surgery for primary RRD. Patients were divided into a recurrence group and a reattachment group. The main outcome measures included causative factors, visual outcomes related to the recurrence of RD, and the perioperative factors most affecting the recurrence of RD.

\section{Results}

After retinal reattachment, we observed RD recurrence after PPV for primary RRD in 42 out of 343 eyes $(12.2 \%)$ during the follow-up period. Most causes of recurrence (69\%) occurred within 6 months of surgery. Univariate analysis showed that the presence of proliferative vitreoretinopathy (PVR) $\geq$ Grade $C$ was significantly associated with recurrent $\mathrm{RD}(P=0.003)$. Logistic regression analysis further showed that a PVR $\geq$ Grade C (odds ratio [OR]: 9.421; 95\% confidence interval [CI]: $2.432-56.39 ; P=0.020$ ) was a significant predictor for the development of recurrent RD. Compared with the reattachment group, the recurrence group exhibited a significant decline in best-corrected visual acuity (BCVA) at the last follow-up visit $(P=0.000)$. Eyes with PVR prior to primary surgery, or at the diagnosis of re-detachment, showed a worse final BCVA.

\section{Conclusions}

Our analysis shows that the predominant risk factor for the recurrence of RD is a PVR $\geq$ Grade C. PVR prior to primary surgery, or at the diagnosis of re-detachment, was also shown to limit the recovery of final visual acuity.

\section{Introduction}

The recurrence of retinal detachment (RD) following rhegmatogenous retinal detachment (RRD) is a relatively common complication that threatens vision and requires a second round of surgery, or even multiple rounds of surgery. Following the development of wide-angle viewing systems, and numerous improvements relating to the equipment used for vitreoretinal surgery, the final anatomical success rates 
for RRD repair can be as high as $93 \%{ }^{[1]}$ However, in some cases, reparative surgery still fails. Previous studies have reported that a range of risk factors are associated with the recurrence of detachment, including proliferative vitreoretinopathy (PVR), ${ }^{[2]}$ inferior breaks, ${ }^{[3]}$ the duration of symptoms, ${ }^{[4]}$ and the

extent of detachment. ${ }^{[5]}$ However, little is known about the relationships between specific risk factors and RRD. There is also some degree of uncertainty with regards to the causes, risk factors, and outcomes of patients with recurrent RD. Understanding the risk factors associated with recurrent RD will help us to predict and prevent this complication. Therefore, the aim of this study was to conduct a retrospective review of a large cohort of patients who received an initial round of pars plana vitrectomy (PPV) surgery for primary RRD, and to investigate the causes, risk factors, and visual outcomes, associated with the recurrence of RD.

\section{Methods}

\section{Study design}

This was a retrospective, long-term, follow-up study. All aspects of the study were conducted in accordance with the Declaration of Helsinki. Informed consent was provided by all of the patients involved using a form approved by the Institutional Review Board (Approval reference number: 2019042). The study included 343 consecutive eyes from 332 patients who received treatment between January 1 , 2015 and January 1, 2018. Patients were recruited if they had received an initial round of vitrectomy surgery for primary RRD, had a postoperative follow-up period exceeding 12 months, and were 18 yearsof-age or older. Patients were excluded if they had received previous rounds of vitreoretinal surgery, had preexisting macular pathological features (e.g., macular hole, age-related macular degeneration, or macular edema), or if they had uveitis, retinal vascular diseases (e.g., diabetic retinopathy or vascular occlusive diseases) or retinal dystrophies.

The main outcome measures included the causative factors and visual outcomes of RDD, and the most predominant perioperative factors associated with the recurrence of RD. For each patient, we examined both eyes. Evaluations included a complete ophthalmological examination, including best-corrected visual acuity (BCVA), intraocular pressure (IOP), slit-lamp biomicroscopy, and optical coherence tomography (OCT) scanning. Images were captured after mydriatic drops had been placed in each eye. In order to evaluate the influence of various factors on the recurrence of retinal detachment, we divided the patients into a recurrence group and a reattachment group.

\section{Data Collection}

We collected a range of demographic and clinical data, including age, gender, medical and ophthalmic history, previous ophthalmic operations, refractive error (high myopia was defined as a preoperative spherical equivalent of $-6 \mathrm{D}$ or more), lens status, macular status (on or off, as determined by preoperative $\mathrm{OCT}$ ), the duration and extent of detachment, the number of breaks, location and type of retinal breaks, 
the presence of posterior vitreous detachment (PVD), the proliferative vitreoretinopathy (PVR) grade (as determined by the Retina Society PVR Classification, $1983^{[6]}$ ), the presence of choroidal detachment, and BCVA at baseline and at follow-up. Irrespective of whether retinectomy was performed or not, all surgeries involved membrane peeling (epiretinal or subretinal), quadrants of endolaser photocoagulation, the use of perfluorocarbon liquid, and combined cataract surgery.

\section{Surgical Technique}

All surgeries were conducted by two experienced retinal surgeons (ZHG and YLZ). Patients underwent elective surgery after a complete physical examination. The time from definitive diagnosis to surgery ranged from 2 to 4 days. We performed a standardized 3-port vitrectomy using a 23- or 25-gauge microincision system. First, we carried out a vitrectomy to release vitreous traction around the retinal breaks and degenerations and then shaved the peripheral vitreous body by scleral indentation. Next, we performed internal drainage of the subretinal fluid to flatten the retina. Endolaser photocoagulation was then performed around the breaks and areas of degeneration. This was followed by a total fluid/gas exchange. We applied silicone oil to all eyes because commercialized medical gas is not available in mainland China. Patients injected with silicone oil were made to adopt and maintain a facedown position for the first 3 weeks after surgery. The silicone oil was removed 3 months later after RRD repair.

\section{Statistical analysis}

All statistical analyses were performed using the Statistical Package for the Social Sciences version 25 (IBM Corp., Armonk, NY, USA). All data were tested for normality prior to analysis using the Shapiro-Wilk test. Qualitative parameters are presented as frequencies and percentages, while quantitative parameters are presented as means and standard deviations. BCVA data were converted to a logarithm of minimal angle of resolution (logMAR) prior to statistical analysis. Differences in categorical data were analyzed using the chi-squared test. Paired t-tests were used to compare visual function between different time points. Multivariate regression analyses were performed to identify potentially confounding parameters. A $p$-value $<0.05$ was considered to be statistically significant.

\section{Results}

\section{General characteristics}

A total of 332 patients (343 eyes) were included in this study. The mean age of the patients was $49.2 \pm$ 10.4 years (range: 18 to 78 years). The mean duration of follow-up was $18.4 \pm 9.1$ months (range: 12 to 43 months). The mean time between symptom onset and surgery was 12.5 days \pm 7.4 days (range: 3 to 110 days). In total, 321 (93.6\%) eyes were phakic and 22 (6.4\%) eyes were pseudophakic. Retinal detachment was macula-on in 134 eyes (39.1\%) and macula-off in 209 eyes (60.9\%). The presence and grade of PVR were assessed prior to the primary procedure: 128 eyes (37.3) showed no signs of PVR, whereas 215 cases with PVR have been classified as followed: 63 eyes with PVR A (29.3\%)凶121 eyes with 
PVR B (56.3\%); 27 eyes with PVR C (12.6\%) and 4 eyes (1.9\%) with PVR D. Patients with low PVR severity (grades $0, A$ or B) collectively as one group, and the other group includes cases with severe PVR (grades C and D). Retinectomy was conducted in 38 eyes $(11.1 \%)$. Following retinal reattachment, the recurrence of RD after PPV for primary RRD was observed in 42 of the 343 eyes (12.2\%) during the follow-up period. The mean time from the recurrence of RD to initial RRD repair was 5.3 months \pm 2.7 months (range: 2 to 13 months). Twenty-nine cases (69\%) were diagnosed with recurrent RD within 6 months of the initial PPV for RRD.

\section{The effect of various perioperative factors on the recurrence of RD}

In order to compare the effects of various factors on the recurrence of RD, we divided our patient population into a recurrence group and reattachment group. A comparison of the perioperative characteristics of the two groups of patients are shown in Table 1. The two groups showed similar perioperative characteristics, except for the presence of PVR $\geq$ Grade C. Univariate analysis demonstrated that the presence of PVR $\geq$ Grade $C$ was a risk factor that was significantly associated with the recurrence of $\operatorname{RD}(P=0.003)$. There were no significant differences between the two groups with regards to age, gender, lens status, the number of breaks, location and type of retinal breaks, the duration of detachment, the extent of retinal detachment, high myopia, macula status, PVD, giant retinal tears, choroidal detachment, the use of perfluorocarbon liquid, retinectomy, and combined cataract surgery. The BCVA did not differ statistically between the groups at baseline $(P=0.342)$. Logistic regression revealed that PVR $\geq$ Grade $C$ (odds ratio [OR]: 9.421; 95\% confidence interval [Cl]: $2.432-56.39, P=0.020$ ) was a significant predictor for the recurrence of RD.

Table 1. A comparison of perioperative characteristics between the recurrence group and reattachment group 


\begin{tabular}{|c|c|c|c|c|}
\hline Parameter & $\begin{array}{l}\text { Overall } \\
(n=343 \\
\text { eyes) }\end{array}$ & $\begin{array}{l}\text { Recurrence group ( } \mathrm{n} \\
=42 \text { eyes) }\end{array}$ & $\begin{array}{l}\text { Reattachment group ( } \mathrm{n} \\
=301 \text { eyes) }\end{array}$ & $\begin{array}{l}P \text { - } \\
\text { value }\end{array}$ \\
\hline \multicolumn{5}{|l|}{ Gender, n (\%) } \\
\hline Male & $\begin{array}{l}172 \\
(50.1)\end{array}$ & $20(47.6)$ & $152(50.5)$ & $0.727^{a}$ \\
\hline Female & $\begin{array}{l}171 \\
(49.9)\end{array}$ & $22(52.4)$ & 149 (49.5) & \\
\hline Age, mean \pm SD & $\begin{array}{l}49.2 \pm \\
10.4\end{array}$ & $49.9 \pm 11.7$ & $48.7 \pm 11.3$ & $0.268^{b}$ \\
\hline \multicolumn{5}{|l|}{ Laterality, n (\%) } \\
\hline Right eye & $\begin{array}{l}184 \\
(53.6)\end{array}$ & $23(54.8)$ & $161(53.5)$ & $0.877^{a}$ \\
\hline Left eye & $\begin{array}{l}159 \\
(46.4)\end{array}$ & $19(45.2)$ & $140(46.5)$ & \\
\hline \multicolumn{5}{|l|}{ Lens status, n (\%) } \\
\hline Phakic & $\begin{array}{l}321 \\
(93.6)\end{array}$ & $38(90.5)$ & $283(94.0)$ & $0.38^{a}$ \\
\hline Pseudophakic & $22(6.4)$ & $4(9.5)$ & $18(6.0)$ & \\
\hline $\begin{array}{l}\text { Detachment duration } \\
\text { (days), mean } \pm \text { SD }\end{array}$ & $\begin{array}{l}12.5 \pm \\
7.4\end{array}$ & $12.1 \pm 9.1$ & $12.9 \pm 8.5$ & $0.607^{b}$ \\
\hline $\begin{array}{l}\text { Quadrants of RRD, mean } \pm \\
\text { SD }\end{array}$ & $\begin{array}{l}2.8 \pm \\
0.79\end{array}$ & $2.7 \pm 0.69$ & $2.8 \pm 0.61$ & $0.478^{b}$ \\
\hline High myopia, n (\%) & $24(7.0)$ & $3(7.1)$ & $21(6.9)$ & $0.968^{a}$ \\
\hline $\begin{array}{l}\text { BCVA (logMAR) at } \\
\text { baseline, mean } \pm S D\end{array}$ & $\begin{array}{l}1.07 \pm \\
0.51\end{array}$ & $1.04 \pm 0.59$ & $1.1 \pm 0.53$ & $0.342^{b}$ \\
\hline \multicolumn{5}{|l|}{ Macular status } \\
\hline On & $\begin{array}{l}134 \\
(39.1)\end{array}$ & $18(42.9)$ & $116(38.5)$ & $0.591^{\mathrm{a}}$ \\
\hline Off & $\begin{array}{l}209 \\
(60.9)\end{array}$ & $24(57.1)$ & 185 (61.5) & \\
\hline PVD (\%) & $\begin{array}{l}238 \\
(69.4)\end{array}$ & $29(69.0)$ & $209(69.4)$ & $0.959^{a}$ \\
\hline Giant retinal tears, n (\%) & $13(3.8)$ & $2(4.8)$ & $11(3.6)$ & $0.725^{\mathrm{a}}$ \\
\hline \multicolumn{5}{|l|}{ Number of breaks, n (\%) } \\
\hline Single breaks & 243 & $\begin{array}{l}31(73.8) \\
\quad \text { Page 6/13 }\end{array}$ & $212(70.4)$ & \\
\hline
\end{tabular}


Multiple breaks

100

(29.2)

$11(26.2)$

89 (29.6)

Type of break, $n$ (\%)

Atrophic holes

131

\38.2】

$17(40.5)$

$114(37.9)$

$0.745^{a}$

Horseshoe tears

212

\61.8》

25 (59.5)

$187(62.1)$

Location of breaks

Superior

217

(63.3)

Inferior

126

(36.7)

$27(64.3)$

$190(63.1)$

$0.884^{a}$

$111(36.9)$

Choroidal detachment

$23(6.7) \quad 3(7.1)$

20 (6.6)

$0.904^{\mathrm{a}}$

PVR $\geq$ Grade C, n (\%)

$31(9.0) \quad 9(21.4)$

$22(7.3)$

$0.003^{a}$

Retinectomy, n (\%)

38 (11.1) $6(14.3)$

32 (10.6)

$0.480^{a}$

Perfluorocarbon liquid, $\mathrm{n}$

$(\%)$

56 (16.3) $8(19.0)$

$48(15.9)$

$0.611^{\mathrm{a}}$

Cataract surgery, $\mathrm{n}(\%)$

$28(8.2) \quad 4(9.5)$

$24(8.0)$

$0.731^{\mathrm{a}}$

Abbreviations: BCVA: best-corrected visual acuity; logMAR: logarithm of minimal angle of resolution; PVD: posterior vitreous detachment; RRD: rhegmatogenous retinal detachment; SD: standard deviation; PVR: proliferative vitreoretinopathy

${ }^{\text {a }} P$ values according to chi-squared tests.

${ }^{\mathrm{b}} P$ values according to t-tests.

$P$ values that are statistically significant $(<0.05)$ are given in bold.

\section{Causes and visual outcomes of patients with recurrent RD}

The main characteristics of patients with recurrent RD are summarized in Table 2. We found that PVR was the main cause of RD recurrence. Overall, 25 eyes (59.5\%) were shown to have posterior PVR, 6 eyes $(14.3 \%)$ had anterior PVR, and 3 eyes $(7.1 \%)$ had a combination of anterior and posterior PVR. In other cases of RD, 5 eyes (11.9\%) showed missed and/or new retinal breaks, 2 cases (4.8\%) were caused by reopening of the primary retinal breaks, and one eye (2.3\%) had a macular hole. In 34 cases, we 
performed another vitrectomy and used silicone oil to repair RD. A further 8 cases received scleral buckling (SB) surgery.

Table 2. Characteristics of the patients with recurrent RD

\begin{tabular}{|ll|}
\hline Parameter & \\
\hline BCVA (logMAR) before a second round surgery & $0.89 \pm 0.47$ \\
\hline BCVA at last follow-up visit & $0.71 \pm 0.22$ \\
\hline Type of RRD, $n$ (\%) & $2354.8)$ \\
\hline Macula-on RD & $19(45.2)$ \\
\hline Macula-off RD & \\
\hline Type of recurrent RD repair surgery, $n$ (\%) & $8(19.0)$ \\
\hline SB & $34(81.0)$ \\
\hline PPV & \\
\hline Gender, $n$ (\%) & $20(47.6)$ \\
\hline Male & $22(52.4)$ \\
\hline Female & $49.9 \pm 11.7$ \\
\hline Age, mean \pm SD & \\
\hline Lens status, $n$ eyes (\%) & $38(90.5)$ \\
\hline Phakic & $24(57.1)$ \\
\hline Pseudophakic & $5.3 \pm 2.7$ \\
\hline Duration between RD repair and RD recurence (months), mean \pm SD & $30(71.4)$ \\
\hline Cause of RD recurrence, $n$ (\%) & $12(28.6)$ \\
\hline PVR & $5(11.9)$ \\
\hline Missed and/or new retinal breaks & $1(2.3)$ \\
\hline Reopening of the primary retinal breaks & \\
\hline Macular hole & \\
\hline Silicone oil, $n(\%)$ & \\
\hline Silicone oil removed & \\
\hline Silicone oil retained & \\
\hline
\end{tabular}


Abbreviations: BCVA, best-corrected visual acuity; logMAR: logarithm of minimal angle of resolution; RD: retinal detachment; PVR: proliferative vitreoretinopathy; SB: scleral buckling; PPV: pars plana vitrectomy;

Silicone oil is usually removed 3 months after primary RRD repair if the retina was attached. In our present series, $12(28.6 \%)$ cases showed re-detachment while the silicone oil was still retained in the eye. Of these 12 patients, 5 presented with an inferior RD, which was treated with a second round of SB surgery. Six cases presented with partial RD caused by PVR and underwent a second vitrectomy and oil procedure, while 3 cases underwent retinectomy. One case developed recurrent RD because of a new retinal break; this condition was treated with vitrectomy and oil. Anatomic reattachment was achieved in all of these patients. Thirty cases (71.4\%) developed recurrent RD following removal of the silicone oil. The mean duration of time to recurrent RD following the removal of silicone oil removal was $2.9 \pm 1.2$ months. With regards to the eyes that developed recurrent RD after the removal of silicone oil, 3 eyes achieved anatomical reattachment following the SB procedure, while a further 24 eyes underwent a second PPV and endolaser photocoagulation. If necessary, we also performed membrane peeling (epiretinal or subretinal), internal limiting membrane peeling, vitrectomy, and silicone oil endotamponade.

Of the 42 patients in which primary PPV failed, we successfully attached the retina in 39 (92.9\%) cases during the second round of surgery. Three cases required a third round of surgery. At the final follow-up visit, the retina had successfully reattached in 41 cases. One of these patients had silicone oil and tamponade at the final follow-up. In one further case, RD persisted, despite the eye being filled with oil. However, this patient did not wish to undergo further surgery because vision was light perception only.

The group of patients experiencing recurrent RD showed a significant reduction in BCVA at the last followup visit when compared with the group of patients without recurrent RD. The mean BCVA at the last follow-up visit was $0.45 \pm 0.32 \log M A R$ in the reattachment group and $0.71 \pm 0.22 \log M A R$ in the recurrence group. Visual acuity at the final follow-up visit was worse in the recurrence group than in the reattachment group $(P=0.000)$. Eyes with PVR prior to primary surgery, or those diagnosed with redetachment, showed a worse BCVA at the last follow-up visit. The mean final LogMAR BCVA was $0.89 \pm$ 0.27 in cases of PVR, whereas the mean final logMAR BCVA was $0.63 \pm 0.29$ in eyes without PVR $(P=$ 0.002).

\section{Discussion}

This study included 343 eyes from 332 patients and revealed that 42 of the patients $(12.2 \%)$ who underwent PPV for RRD went on to subsequently experience re-detachment. This result concurred well with the findings of previous studies on primary RRD. ${ }^{[7,8]}$ Most cases of postoperative re-detachment $(69 \%)$ were diagnosed within 6 months of the initial surgery for RRD. This result is lower than that reported by previous studies; for example, one study reported that $97.9 \%$ of cases with recurrent RD were diagnosed within 6 months of the initial vitrectomy. ${ }^{[9]}$ This inconsistency may be related to the frequency of follow-up visits. Overall, we found that the overall final success rate was $97.6 \%$; these findings were comparable to those reported by previous studies. ${ }^{[10,11]}$ 
Previous studies have reported that a wide variety of risk factors are associated with the recurrence of RD after RRD repair, including proliferative vitreoretinopathy (PVR) ${ }^{[12]}$ inferior breaks, ${ }^{[3]}$ the duration of preoperative symptoms prior to surgery, ${ }^{[13]}$ lens status, ${ }^{[14]}$ vitreous hemorrhage, ${ }^{[15]}$ undetectable retinal tear, $^{[16]}$ the extent of detachment, ${ }^{[5]}$ high myopia, ${ }^{[17]}$ hypotonia, ${ }^{[18]}$ and the presence of multiple breaks. ${ }^{[10]}$ Unlike these previous studies, our present study failed to identify significant differences between groups of patients with recurrent and reattached RD with regards to age, gender, lens status, the number of breaks, the duration of detachment, location and type of retinal breaks, the extent of retinal detachment, high myopia, macula status, PVD, giant retinal tears, choroidal detachment, the use of perfluorocarbon liquid, retinectomy, and combined cataract surgery. However, we identified that PVR $\geq$ Grade $C$ was significantly associated with recurrent RD. Logistic regression analysis further showed that PVR $\geq$ Grade $\mathrm{C}$ was a significant independent risk factor for the recurrence of RD. Previous studies have shown that PVR is the most important risk factor in RRD at presentation. ${ }^{[15,19]}$ However, in the present study, we confirmed that PVR is the best predictive factor for the failure of PPV in RRD.

We analyzed the causes of retinal detachment after vitrectomy and found that PVR was the most predominant factor responsible for the primary failure of attachment surgery. We also found that the main causes of recurrent RD were the development of postoperative PVR and the generation of new retinal breaks. Overall, our data showed that $81.0 \%$ of recurrent RD was caused by the development of postoperative PVR, a condition that is also known to be an important cause of new retinal breaks during the postoperative period. Anterior PVR, or posterior PVR, can both lead to the recurrence of RD via tractional mechanisms or a combination of tractional and rhegmatogenous mechanisms. ${ }^{[11]}$ PVR can cause contraction of the peri-retinal cellular membranes, and can therefore cause new retinal breaks, or cause old retinal breaks to re-open. PVR can also cause distortion of the retina, resulting in sequalae that are detrimental to vision. [20]

In this study, we assessed the visual acuity of patients undergoing secondary surgery for redetachment and found that surgery only resulted in limited improvement in terms of visual acuity. Even if anatomical attachment was successful, there was a significant deterioration in final visual acuity. It is noteworthy that the development of PVR led to low visual acuity, irrespective of whether PVR developed preoperatively or postoperatively. The development of PVR not only affected the success rates of primary surgery, but also caused disruption to retinal function. The presence of PVR is an important factor underlying the reduction in final BCVA. ${ }^{[21]}$ Despite successful anatomical reattachment, a previous study reported that most patients with primary and secondary PPV, with PVR grades of $C$ and D, had a visual acuity of $20 / 200$, or worse. ${ }^{[22]}$ Other factors are known to affect the loss of vision, including glaucoma, silicone oil and the development of cataracts postoperatively, so we cannot completely rule out the possibility of visual impairment caused by cataract, glaucoma and silicone oil.

When patients experienced the recurrence of RD, the most common surgical intervention was secondary PPV. Nevertheless, an additional SB procedure can often be used in cases of inferior re-detachment. In our present study, we experienced 5 cases with an inferior quadrant re-detachment. These were treated by 
silicon oil filling and SB. In all 5 cases, reattachment was successful. We found that it was safe to carry out supplementary SB in an eye filled with silicone oil in the presence of inferior retinal breaks.

There were several limitations to our study that need to be considered. First, the retrospective design of this study may have led to recruitment bias and overestimated the incidence of recurrent RD. It is also possible that cases with favorable visual acuity may have been excluded from our study because of the short follow-up period. Second, we only analyzed a relatively small number of patients. Our findings should now be confirmed by conducting prospective studies on a much larger scale. Third, other factors are known to affect the loss of vision, such as cataract and glaucoma; these conditions are known to be related to surgery and the use of silicone oil tamponade.

\section{Conclusions}

In summary, we demonstrated that the presence of PVR $\geq$ Grade $C$ represents a significant independent risk factor for the recurrence of RD. The most predominant causes of recurrent RD were the development of postoperative PVR and the generation of new retinal breaks. The development of PVR, either at initial presentation, or on the diagnosis of re-detachment, led to reduced levels of visual acuity, even after the retina had been completely reattached. In order to avoid the recurrence of postoperative RD, it is important to carefully undertake a comprehensive preoperative examination to avoid the omission of retinal breaks and degeneration. It is also important to select the most appropriate surgical methods to suit different situations. It is also important to take a delicate approach during operations so that further damage to the retina can be avoided, such as iatrogenic hiatus and the formation of postoperative PVR. Long-term follow-up is essential following RRD repair. Our findings indicate that surgeons should pay particular attention to the recovery of primary retinal breaks.

\section{Abbreviations}

RD: retinal detachment; RRD: rhegmatogenous retinal detachment; PVR: proliferative vitreoretinopathy; PPV: pars plana vitrectomy; BCVA: best-corrected visual acuity; IOP: intraocular pressure; OCT: optical coherence tomography; PVD: posterior vitreous detachment; logMAR: logarithm of minimal angle of resolution

\section{References}

1. Quiram PA, Gonzales CR, Hu W et al (2006) Outcomes of vitrectomy with inferior retinectomy in patients with recurrent rhegmatogenous retinal detachments and proliferative vitreoretinopathy. Ophthalmology 113(11):2041-2047

2. Tseng W, Cortez RT, Ramirez G et al (2004) Prevalence and risk factors for proliferative vitreoretinopathy in eyes with rhegmatogenous retinal detachment but no previous vitreoretinal surgery. Am J Ophthalmol 137(6):1105-1115 
3. Goto T, Nakagomi T, lijima H (2013) A comparison of the anatomic successes of primary vitrectomy for rhegmatogenous retinal detachment with superior and inferior breaks. Acta Ophthalmol 91(6):552-556

4. Enders P, Schick T, Kemper C,et al (2017) Effect of Symptom Duration until Surgery on First-Year Outcome in Pseudophakic Primary Rhegmatogenous Retinal Detachment. Ophthalmologica 237(2):73-77

5. Heimann H, Zou X, Jandeck C et al (2006) Primary vitrectomy for rhegmatogenous retinal detachment: an analysis of 512 cases. Graefes Arch Clin Exp Ophthalmol 244(1):69-78

6. The classification of (1983) retinal detachment with proliferative vitreoretinopathy. Ophthalmology 90(2):121-125

7. Regler R, Sachs HG, Hillenkamp J et al (2009) Long-term evaluation of anatomic and functional results after complicated retinal detachment treated with pars plana vitrectomy and heavy silicone oil tamponade. Klin Monbl Augenheilkd 226(9):707-712

8. Ozdek S, Yuksel N, Gurelik G et al (2011) High-density silicone oil as an intraocular tamponade in complex retinal detachments. Can J Ophthalmol 46(1):51-55

9. Lee E, El Housseini Z, Steel DH et al (2014) An analysis of the outcomes for patients with failed primary vitrectomy for rhegmatogenous retinal detachment. Graefes Arch Clin Exp Ophthalmol 252(11):1711-1716

10. Ambiya V, Rani PK, Narayanan R et al (2018) Outcomes of Recurrent Retinal Detachment Surgery following Pars Plana Vitrectomy for Rhegmatogenous Retinal Detachment. Semin Ophthalmol 33(5):657-663

11. Beuste T, Rebollo O, Parrat E et al: RECURRENCES OF RETINAL DETACHMENT AFTER RETINECTOMY: Causes and Outcomes. Retina 2019

12. Adelman RA, Parnes AJ, Michalewska Z et al (2014) European Vitreo-Retinal Society Retinal Detachment Study G: Clinical variables associated with failure of retinal detachment repair: the European vitreo-retinal society retinal detachment study report number 4 . Ophthalmology 121(9):1715-1719

13. Uzel MM, Citirik $M$, Ilhan $C$ et al: The impact of duration on the recurrence of rhegmatogenous retinal detachment: optimal cutoff value. Int Ophthalmol 2018

14. Caiado RR, Magalhaes $O \mathrm{Jr}$, Badaro E et al (2015) Effect of lens status in the surgical success of 23gauge primary vitrectomy for the management of rhegmatogenous retinal detachment: the Pan American Collaborative Retina Study (PACORES) group results. Retina 35(2):326-333

15. Wickham L, Bunce C, Wong D (2011) Retinal detachment repair by vitrectomy: simplified formulae to estimate the risk of failure. $\mathrm{Br} \mathrm{J}$ Ophthalmol 95(9):1239-1244

16. Pournaras CJ, Kapetanios AD (2003) Primary vitrectomy for pseudophakic retinal detachment: a prospective non-randomized study. Eur J Ophthalmol 13(3):298-306

17. He Y, Zeng S, Zhang Y et al (2018) Risk Factors for Retinal Redetachment After Silicone Oil Removal: A Systematic Review and Meta-Analysis. Ophthalmic Surg Lasers Imaging Retina 49(6):416-424 
18. La Heij EC, Hendrikse F, Kessels AG (2001) Results and complications of temporary silicone oil tamponade in patients with complicated retinal detachments. Retina 21(2):107-114

19. Enders P, Schick T, Schaub F et al (2017) Risk of Multiple Recurring Retinal Detachment after Primary Rhegmatogenous Retinal Detachment Repair. Retina 37(5):930-935

20. Sethi CS, Lewis GP, Fisher SK et al (2005) Glial remodeling and neural plasticity in human retinal detachment with proliferative vitreoretinopathy. Invest Ophthalmol Vis Sci 46(1):329-342

21. de Silva DJ, Kwan A, Bunce $C$ et al (2008) Predicting visual outcome following retinectomy for retinal detachment. Br J Ophthalmol 92(7):954-958

22. Uney GO, Acar MA, Unlu N et al (2016) Pars plana vitrectomy with silicone oil tamponade in failed scleral buckle surgery. Can J Ophthalmol 51(5):331-335 